\title{
A Novel Guidance Law with Line-of-Sight Acceleration Feedback for Missiles against Maneuvering Targets
}

\author{
Kemao $\mathrm{Ma}^{1}$ and Xiaoyu Zhang ${ }^{2}$ \\ ${ }^{1}$ Control and Simulation Center, School of Astronautics, Harbin Institute of Technology, Harbin 150080, China \\ ${ }^{2}$ College of Automation, Harbin Engineering University, Harbin 150001, China \\ Correspondence should be addressed to Xiaoyu Zhang; zhangxiaoyu@hrbeu.edu.cn
}

Received 28 July 2014; Accepted 15 November 2014; Published 25 November 2014

Academic Editor: Sabri Arik

Copyright (c) $2014 \mathrm{~K}$. Ma and X. Zhang. This is an open access article distributed under the Creative Commons Attribution License, which permits unrestricted use, distribution, and reproduction in any medium, provided the original work is properly cited.

\begin{abstract}
Terminal guidance law design and its implementation are considered for homing missiles against maneuvering targets. The lateral acceleration dynamics are taken into account in the design. In the guidance law design, the line-of-sight acceleration signals are incorporated into the acceleration reference signals to compensate for the targets' maneuvers. Then the commanded accelerations are designed and the convergent tracking of the lateral accelerations to these signals is proven theoretically. In the guidance implementation, a linear high-gain differentiator is used to estimate the line-of-sight rates and the line-of-sight acceleration signals. To avoid the magnifying effects of higher order differentiation, a practical design of commanded accelerations is given to realize approximate tracking of the lateral accelerations to the given reference signals. Simulation is conducted for both cases with and without measurement noises. The simulation results justify the feasibility of the design and the implementation.
\end{abstract}

\section{Introduction}

For terminal guidance laws of the homing missiles against maneuvering targets, two of the most important factors affecting the guidance precision are the targets' maneuvers and the lateral acceleration dynamics of the missiles [1]. If these two factors are ignored in mathematical performance analysis, then the conventional proportional navigation guidance (PNG) laws, which provide for missiles lateral acceleration commands proportional to the line-of-sight rates, are optimal in the sense that both the miss distance and control efforts are accounted for in the performance cost functional [2]. This result motivated many guidance laws based on PNG. For a maneuvering target, an augmented PNG law consists of conventional PNG and a compensation term for the target's maneuvering acceleration. Thus, the target's maneuver is canceled out in the relative kinematics of the missile and the target, and the performance of PNG against a nonmaneuvering target is recovered. Since the target's maneuver is generally unknown, the remaining problem is how to estimate the target's maneuver in guidance law implementation. For a seeker with bearing only measurement, such as an infrared seeker, the acceleration components of the target's maneuver are not observable. Therefore, Kalman filter technique, as well as conventional state observer technique, would be practical only if an appropriate maneuvering model for the target's maneuver was designed for the observability condition to be met [3]. By extending the observer states, however, extended state observer technique can be used to estimate the target's maneuver without making any a priori assumptions on target's maneuver $[4,5]$. In fact, extending the observer states is equivalent to a constant acceleration (CA) model being adopted, and the discrepancy between the actual target's maneuver and the output of the CA model is treated as an uncertain term in the observer error dynamics and is suppressed via high-gain feedback. Thus no further delicate models of the target's maneuver are necessary.

The compensation for the targets' maneuvers is a feedforward method. The targets' maneuvers can also be treated in a feedback paradigm. In this case, the maneuvers of the targets are thought of as external disturbance inputs for the guidance systems, and feedback control methods with good disturbance rejection or attenuation can be used to design the guidance laws. Indeed, based on sliding mode control, 
various guidance laws are designed in which a switching term is added to the PNG term [6,7]. The sliding mode guidance laws are nonsmooth, which may result in theoretical difficulties in guidance law implementation when the lateral acceleration dynamics of the missiles are considered.

Both theoretical analysis and numerical simulation show that the lateral acceleration dynamics of the missiles can induce miss distance, especially when the time constants are large. There are two approaches to solving this problem. One approach is to improve the response characteristics of the lateral acceleration dynamics by adopting novel techniques such as lateral jets [8], and the guidance and control systems are in a decoupled structure and designed in separately. The introduction of the lateral jets endows the missiles with features of heterogeneous multiple actuators and substantial uncertainties due to the side jet interaction effects [9], which bring potential difficulties in the design of the guidance and control systems. The other approach is to conduct an integrated design of guidance and control [10]. In an integrated design, the guidance, control, and guidance information estimation are treated as a whole coupled system for which certain performance index is to be optimized. The integrated system is quite complex with multiple constraints compared with the guidance system or the control system in a decoupled structure, and there are still difficulties to be tackled in both theory and application.

In this paper, a novel guidance law is designed for missiles against maneuvering targets. In the guidance law, the target's maneuvers are compensated for via incorporating the lineof-sight accelerations into the guidance commands, instead of constructing a filter or an observer. Also incorporated into the commands are the dynamics of the missiles via a robust control design. The idea is to design the guidance and control separately and then to redesign the lateral acceleration commands issued by the guidance laws by incorporating the closed-loop lateral acceleration dynamics into the commands. By doing so, a high guidance precision is reached. The rest of the paper is organized as follows. In Section 2, the three-dimensional relative kinematics of a missile and its maneuvering target are given, and the problem of terminal guidance law design is formulated. In Section 3, a guidance law is designed via incorporating into guidance commands the line-of-sight accelerations and the lateral acceleration dynamics of the missile. In the following section, guidance law implementation is considered, and a practical design of the acceleration commands is given. In Section 5, numerical simulation is conducted. The conclusion is given in Section 6.

\section{Problem Formulation}

We assume the terminal guidance scenario of a missile against a maneuvering air target. It is convenient to describe the kinematics of the relative motion between the missile and its target in the line-of-sight coordinate system, denoted by $O x_{L} y_{L} z_{L}$ and shown in Figure 1. The origin $O$ of $O x_{L} y_{L} z_{L}$ is set to the mass center of the missile (denoted by $M$ ); the axis $O x_{L}$ is aligned with the line-of-sight (denoted by LOS)

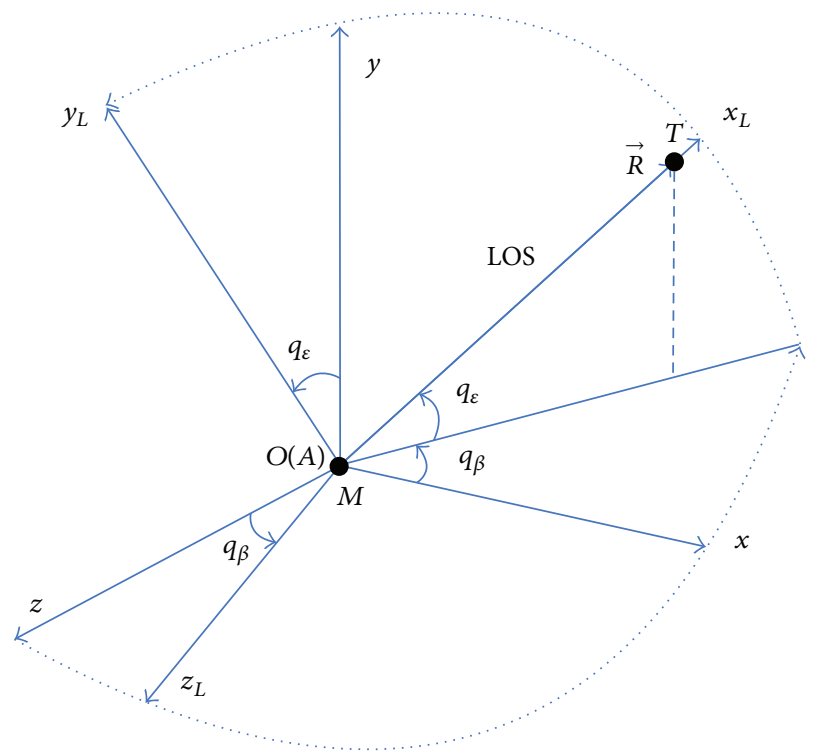

FIgURE 1: Orientation of $O x_{L} y_{L} z_{L}$ with respect to $A x y z$.

and the half-line originated from $O$ and pointing to the target (denoted by $T$ ); the axis $O y_{L}$ is in the vertical plane, pointing upward and normal to $O x_{L}$; and the axis $O z_{L}$ is normal to both $O x_{L}$ and $O y_{L}$ with its positive direction decided by the right-hand rule. We take the earth-fixed coordinate system $A x y z$ as an inertial reference. $O x_{L} y_{L} z_{L}$ can be obtained through a translation of $A x y z$, followed by two consecutive counterclockwise rotations, first with an Euler angle $q_{\beta}$ with respect to $A y$ and second with an Euler angle $q_{\varepsilon}$ with respect to $O z_{L}$, as shown in Figure 1, where, for clarity of illustration, the origin $A$ of $A x y z$ is already translated to coincide with the origin $O$ of $O x_{L} y_{L} z_{L}$, and the rotations are indicated with dotted curved arrows. Thus, the orientation of $O x_{L} y_{L} z_{L}$ with respect to $A x y z$ is characterized by $q_{\varepsilon}$ and $q_{\beta}$, known as lineof-sight angles. The relative range vector, for example, the radius vector of the target in $O x_{L} y_{L} z_{L}$ originating from $M$ towards $T$ along LOS, is denoted by $\vec{R}$, and its magnitude, the relative range, is denoted by $R$.

Next, we construct the mathematical description of the relative motion of the missile and the target in $O x_{L} y_{L} z_{L}$. Denote the respective unit vectors of $O x_{L}, O y_{L}$, and $O z_{L}$ by $\vec{i}_{L}, \vec{j}_{L}$, and $\vec{k}_{L}$, and we have

$$
\vec{R}=R \vec{i}_{L}+0 \vec{j}_{L}+0 \vec{k}_{L}
$$

Denote the unit vectors of $A x, A y$, and $A z$ by $\vec{i}, \vec{j}$, and $\vec{k}$, respectively. Figure 1 shows that

$$
\left[\begin{array}{c}
\vec{i}_{L} \\
\vec{j}_{L} \\
\vec{k}_{L}
\end{array}\right]=L\left(q_{\varepsilon}, q_{\beta}\right)\left[\begin{array}{c}
\vec{i} \\
\vec{j} \\
\vec{k}
\end{array}\right],
$$


where

$$
\begin{aligned}
L\left(q_{\varepsilon}, q_{\beta}\right) & =\left[\begin{array}{ccc}
\cos q_{\varepsilon} & \sin q_{\varepsilon} & 0 \\
-\sin q_{\varepsilon} & \cos q_{\varepsilon} & 0 \\
0 & 0 & 1
\end{array}\right]\left[\begin{array}{ccc}
\cos q_{\beta} & 0 & -\sin q_{\beta} \\
0 & 1 & 0 \\
\sin q_{\beta} & 0 & \cos q_{\beta}
\end{array}\right] \\
& =\left[\begin{array}{ccc}
\cos q_{\varepsilon} \cos q_{\beta} & \sin q_{\varepsilon} & -\cos q_{\varepsilon} \sin q_{\beta} \\
-\sin q_{\varepsilon} \cos q_{\beta} & \cos q_{\varepsilon} & \sin q_{\varepsilon} \sin q_{\beta} \\
\sin q_{\beta} & 0 & \cos q_{\beta}
\end{array}\right] .
\end{aligned}
$$

Consider the two consecutive rotations shown in Figure 1, and we have the expression of the angular velocity of $O x_{L} y_{L} z_{L}$ with respect to $A x y z$ as follows:

$$
\begin{aligned}
\vec{\omega} & =\dot{q}_{\beta} \vec{j}+\dot{q}_{\varepsilon} \vec{k}_{L} \\
& =\dot{q}_{\beta} \sin q_{\varepsilon} \vec{i}_{L}+\dot{q}_{\beta} \cos q_{\varepsilon} \vec{j}_{L}+\dot{q}_{\varepsilon} \vec{k}_{L}
\end{aligned}
$$

where the latter equality is obtained using (2). The relative velocity between the missile and the target is

$$
\begin{aligned}
\dot{\vec{R}} & =\dot{R} \vec{i}_{L}+\vec{\omega} \times R \vec{i}_{L} \\
& =\dot{R} \vec{i}_{L}+R \dot{q}_{\varepsilon} \vec{j}_{L}-R \dot{q}_{\beta} \cos q_{\varepsilon} \vec{k}_{L},
\end{aligned}
$$

which further leads to

$$
\begin{aligned}
\ddot{\vec{R}}= & \ddot{R} \vec{i}_{L}+\dot{R} \dot{\vec{i}}_{L}+\left(\dot{R} \dot{q}_{\varepsilon}+R \ddot{q}_{\varepsilon}\right) \vec{j}_{L}+R \dot{q}_{\varepsilon} \dot{\vec{j}}_{L} \\
& -\left(\dot{R} \dot{q}_{\beta} \cos q_{\varepsilon}+R \ddot{q}_{\beta} \cos q_{\varepsilon}-R \dot{q}_{\beta} \dot{q}_{\varepsilon} \sin q_{\varepsilon}\right) \vec{k}_{L} \\
& -R \dot{q}_{\beta} \cos q_{\varepsilon} \dot{\vec{k}}_{L},
\end{aligned}
$$

where

$$
\begin{aligned}
\dot{\vec{i}}_{L} & =\vec{\omega} \times \vec{i}_{L} \\
& =\dot{q}_{\varepsilon} \vec{j}_{L}-\dot{q}_{\beta} \cos q_{\varepsilon} \vec{k}_{L}, \\
\dot{\vec{j}}_{L} & =\vec{\omega} \times \vec{j}_{L} \\
& =-\dot{q}_{\varepsilon} \vec{i}_{L}+\dot{q}_{\beta} \sin q_{\varepsilon} \vec{k}_{L}, \\
\dot{\vec{k}}_{L} & =\vec{\omega} \times \vec{k}_{L} \\
& =\dot{q}_{\beta} \cos q_{\varepsilon} \vec{i}_{L}-\dot{q}_{\beta} \sin q_{\varepsilon} \vec{j}_{L} .
\end{aligned}
$$

Substitute these expressions into (6), and we have

$$
\begin{aligned}
\ddot{\vec{R}}= & \left(\ddot{R}-R \dot{q}_{\varepsilon}^{2}-R \dot{q}_{\beta}^{2} \cos ^{2} q_{\varepsilon}\right) \vec{i}_{L} \\
+ & \left(2 \dot{R} \dot{q}_{\varepsilon}+R \ddot{q}_{\varepsilon}+R \dot{q}_{\beta}^{2} \sin q_{\varepsilon} \cos q_{\varepsilon}\right) \vec{j}_{L} \\
+ & \left(-2 \dot{R} \dot{q}_{\beta} \cos q_{\varepsilon}+R \dot{q}_{\varepsilon} \dot{q}_{\beta} \sin q_{\varepsilon}-R \ddot{q}_{\beta} \cos q_{\varepsilon}\right. \\
& \left.+R \dot{q}_{\varepsilon} \dot{q}_{\beta} \sin q_{\varepsilon}\right) \vec{k}_{L} .
\end{aligned}
$$

Denote the projections of the target's acceleration on $O x_{L}$, $O y_{L}$, and $O z_{L}$ by $a_{T r}, a_{T \varepsilon}$, and $a_{T \beta}$, respectively, and denote the projections of the missile's acceleration by $a_{M r}, a_{M \varepsilon}$, and $a_{M \beta}$, respectively. Account for the contributions to $\ddot{\vec{R}}$ of these projections, and we have

$$
\ddot{\vec{R}}=\left(a_{T r}-a_{M r}\right) \vec{i}_{L}+\left(a_{T \varepsilon}-a_{M \varepsilon}\right) \vec{j}_{L}+\left(a_{T \beta}-a_{M \beta}\right) \vec{k}_{L} .
$$

Equating (8) to (9) gives the following mathematical description of the relative motion between the missile and its target:

$$
\begin{gathered}
\ddot{R}=R \dot{q}_{\varepsilon}^{2}+R \dot{q}_{\beta}^{2} \cos ^{2} q_{\varepsilon}+a_{T r}-a_{M r}, \\
\ddot{q}_{\varepsilon}=-\frac{2 \dot{R}}{R} \dot{q}_{\varepsilon}-\dot{q}_{\beta}^{2} \sin q_{\varepsilon} \cos q_{\varepsilon}+\frac{a_{T \varepsilon}-a_{M \varepsilon}}{R}, \\
\ddot{q}_{\beta}=-\frac{2 \dot{R}}{R} \dot{q}_{\beta}+2 \dot{q}_{\varepsilon} \dot{q}_{\beta} \tan q_{\varepsilon}-\frac{a_{T \beta}-a_{M \beta}}{R \cos q_{\varepsilon}} .
\end{gathered}
$$

In (11) and (12), the target's acceleration components $a_{T \varepsilon}$ and $a_{T \beta}$ are unknown and can be thought of as external disturbances. The acceleration components $a_{M \varepsilon}$ and $a_{M \beta}$ are provided by the missile acceleration dynamics which can be modeled as follows:

$$
\begin{aligned}
& \ddot{a}_{M \varepsilon}=-2 \zeta \omega_{n} \dot{a}_{M \varepsilon}-\omega_{n}^{2} a_{M \varepsilon}+\omega_{n}^{2} a_{M \varepsilon c}+\Delta_{\varepsilon}\left(a_{M \varepsilon}, \dot{a}_{M \varepsilon}, t\right), \\
& \ddot{a}_{M \beta}=-2 \zeta \omega_{n} \dot{a}_{M \beta}-\omega_{n}^{2} a_{M \beta}+\omega_{n}^{2} a_{M \beta c}+\Delta_{\beta}\left(a_{M \beta}, \dot{a}_{M \beta}, t\right),
\end{aligned}
$$

where the dynamics are modeled as second-order linear dynamics with damping ratio $\zeta>0$ and natural frequency $\omega_{n}>0$, and $a_{M \varepsilon c}$ and $a_{M \beta c}$ are guidance commands to be designed. The differences of the second-order models from the real dynamics of the missile are lumped into the uncertainties $\Delta_{\varepsilon}\left(a_{M \varepsilon}, \dot{a}_{M \varepsilon}, t\right)$ and $\Delta_{\beta}\left(a_{M \beta}, \dot{a}_{M \beta}, t\right)$ which satisfy, uniformly in $t$, the following inequalities:

$$
\begin{aligned}
& \left|\Delta_{\varepsilon}\left(a_{M \varepsilon}, \dot{a}_{M \varepsilon}, t\right)\right| \leqslant \bar{a}_{M}, \\
& \left|\Delta_{\beta}\left(a_{M \beta}, \dot{a}_{M \beta}, t\right)\right| \leqslant \bar{a}_{M},
\end{aligned}
$$

where $\bar{a}_{M}$ is a known constant.

Classical guidance theory shows that if the line-of-sight rates $\dot{q}_{\varepsilon}$ and $\dot{q}_{\beta}$ are convergent to zero, then a satisfactory miss distance can be guaranteed. Thus the guidance law design problem can be formulated as follows: with the existence of the external disturbances $a_{T \varepsilon}$ and $a_{T \beta}$ and uncertainties $\Delta_{\varepsilon}\left(a_{M \varepsilon}, \dot{a}_{M \varepsilon}, t\right)$ and $\Delta_{\beta}\left(a_{M \beta}, \dot{a}_{M \beta}, t\right)$ satisfying (14), design $a_{M \varepsilon c}$ and $a_{M \beta c}$ in (13) such that $a_{M \varepsilon}$ and $a_{M \beta}$ in (11) and (12) can make $\dot{q}_{\varepsilon}$ and $\dot{q}_{\beta}$ convergent to zero.

\section{Guidance Law Design Based on Line-of- Sight Acceleration Feedback}

If the following equalities hold

$$
\begin{aligned}
& a_{M \varepsilon}=a_{T \varepsilon}-2 \dot{R} \dot{q}_{\varepsilon}-R \dot{q}_{\beta}^{2} \sin q_{\varepsilon} \cos q_{\varepsilon}+k R \dot{q}_{\varepsilon}, \\
& a_{M \beta}=a_{T \beta}+2 \dot{R} \dot{q}_{\beta} \cos q_{\varepsilon}-2 R \dot{q}_{\varepsilon} \dot{q}_{\beta} \sin q_{\varepsilon}-k R \dot{q}_{\beta} \cos q_{\varepsilon},
\end{aligned}
$$


where $k$ is a positive constant, then (11) and (12) become

$$
\begin{aligned}
& \ddot{q}_{\varepsilon}=-k \dot{q}_{\varepsilon}, \\
& \ddot{q}_{\beta}=-k \dot{q}_{\beta} .
\end{aligned}
$$

Equations (16) guarantee the exponential convergence of the line-of-sight rates, and the convergence rates are determined by parameter $k$. Generally speaking, equalities (15) do not hold. However, if commanded acceleration components $a_{M \varepsilon c}$ and $a_{M \beta c}$ are designed such that the left-hand sides of (15), $a_{M \varepsilon}$ and $a_{M \beta}$, can track the respective right-hand sides, then the dynamics of line-of-sight rates (16) will hold in an approximation sense. Thus the right-hand sides of (15) should be incorporated into $a_{M \varepsilon c}$ and $a_{M \beta c}$ as reference signals for $a_{M \varepsilon}$ and $a_{M \beta}$ to track. To deal with the unknown terms $a_{T \varepsilon}$ and $a_{T \beta}$, we rewrite (11) and (12) as

$$
\begin{gathered}
R \ddot{q}_{\varepsilon}+a_{M \varepsilon}=a_{T \varepsilon}-2 \dot{R} \dot{q}_{\varepsilon}-R \dot{q}_{\beta}^{2} \sin q_{\varepsilon} \cos q_{\varepsilon}, \\
a_{M \beta}-R \ddot{q}_{\beta} \cos q_{\varepsilon}=a_{T \beta}+2 \dot{R} \dot{q}_{\beta} \cos q_{\varepsilon}-2 R \dot{q}_{\varepsilon} \dot{q}_{\beta} \sin q_{\varepsilon} .
\end{gathered}
$$

Comparing the right-hand sides of (15) with those of (17) suggests taking the reference signals in the form of

$$
\begin{aligned}
& a_{M \varepsilon r}=a_{M \varepsilon}+R \ddot{q}_{\varepsilon}+k R \dot{q}_{\varepsilon}, \\
& a_{M \beta r}=a_{M \beta}-R \ddot{q}_{\beta} \cos q_{\varepsilon}-k R \dot{q}_{\beta} \cos q_{\varepsilon},
\end{aligned}
$$

based on which the commanded accelerations are in the form of

$$
\begin{aligned}
& a_{M \varepsilon c}=a_{M \varepsilon r}+K_{\varepsilon}, \\
& a_{M \beta c}=a_{M \beta r}+K_{\beta},
\end{aligned}
$$

where $K_{\varepsilon}$ and $K_{\beta}$ are yet to be designed. In (18) line-ofsight accelerations, $\ddot{q}_{\varepsilon}$ and $\ddot{q}_{\beta}$, are used to compensate for the target's unknown maneuvers. The compensation effect depends on the tracking of $a_{M \varepsilon}$ and $a_{M \beta}$ to the respective right-hand sides of (15). In the sequel, we design $K_{\varepsilon}\left(a_{M \varepsilon}, \dot{a}_{M \varepsilon}\right)$ and $K_{\beta}\left(a_{M \beta}, \dot{a}_{M \beta}\right)$ to guarantee the tracking, as well as to account for the uncertainties in (13).

Define the tracking error vectors as

$$
\begin{aligned}
e_{\varepsilon} & =\left[\begin{array}{ll}
e_{\varepsilon 1} & e_{\varepsilon 2}
\end{array}\right]^{T} \\
& =\left[\begin{array}{ll}
a_{M \varepsilon}-a_{M \varepsilon r} & \dot{a}_{M \varepsilon}-\dot{a}_{M \varepsilon r}
\end{array}\right]^{T}, \\
e_{\beta} & =\left[\begin{array}{ll}
e_{\beta 1} & e_{\beta 2}
\end{array}\right]^{T} \\
& =\left[\begin{array}{ll}
a_{M \beta}-a_{M \beta r} & \dot{a}_{M \beta}-\dot{a}_{M \beta r}
\end{array}\right]^{T},
\end{aligned}
$$

and, according to (13), we have the error dynamics as follows:

$$
\begin{aligned}
& \dot{e}_{\varepsilon}=A e_{\varepsilon}+B\left(a_{M \varepsilon c}-a_{M \varepsilon r}+f_{\varepsilon}+\frac{1}{\omega_{n}^{2}} \Delta_{\varepsilon}\left(a_{M \varepsilon}, \dot{a}_{M \varepsilon}, t\right)\right), \\
& \dot{e}_{\beta}=A e_{\beta}+B\left(a_{M \beta c}-a_{M \beta r}+f_{\beta}+\frac{1}{\omega_{n}^{2}} \Delta_{\beta}\left(a_{M \beta}, \dot{a}_{M \beta}, t\right)\right),
\end{aligned}
$$

where

$$
\begin{gathered}
f_{\varepsilon}=-\frac{2 \zeta \dot{a}_{M \varepsilon r}}{\omega_{n}}-\frac{\ddot{a}_{M \varepsilon r}}{\omega_{n}^{2}}, \\
f_{\beta}=-\frac{2 \zeta \dot{a}_{M \beta r}}{\omega_{n}}-\frac{\ddot{a}_{M \beta r}}{\omega_{n}^{2}}, \\
A=\left[\begin{array}{cc}
0 & 1 \\
-\omega_{n}^{2} & -2 \zeta \omega_{n}
\end{array}\right], \quad B=\left[\begin{array}{c}
0 \\
\omega_{n}^{2}
\end{array}\right] .
\end{gathered}
$$

Substitute (19) into (21), and we have

$$
\begin{aligned}
& \dot{e}_{\varepsilon}=A e_{\varepsilon}+B\left(K_{\varepsilon}+f_{\varepsilon}+\frac{1}{\omega_{n}^{2}} \Delta_{\varepsilon}\left(a_{M \varepsilon}, \dot{a}_{M \varepsilon}, t\right)\right), \\
& \dot{e}_{\beta}=A e_{\beta}+B\left(K_{\beta}+f_{\beta}+\frac{1}{\omega_{n}^{2}} \Delta_{\beta}\left(a_{M \beta}, \dot{a}_{M \beta}, t\right)\right) .
\end{aligned}
$$

Since $\zeta>0$ and $\omega_{n}>0, A$ in (24) is a Hurwitz matrix. Therefore, for any $Q>0$, there is a $P>0$, such that the following Lyapunov equation holds:

$$
A^{T} P+P A=-Q .
$$

For error dynamics (25), define the Lyapunov function candidate as $V=e_{\varepsilon}^{T} P e_{\varepsilon}+e_{\beta}^{T} P e_{\beta}$, and we have

$$
\begin{aligned}
\dot{V}= & e_{\varepsilon}^{T}\left(A^{T} P+P A\right) e_{\varepsilon}+e_{\beta}^{T}\left(A^{T} P+P A\right) e_{\beta} \\
& +2 e_{\varepsilon}^{T} P B\left(K_{\varepsilon}+f_{\varepsilon}+\frac{1}{\omega_{n}^{2}} \Delta_{\varepsilon}\left(a_{M \varepsilon}, \dot{a}_{M \varepsilon}, t\right)\right) \\
& +2 e_{\beta}^{T} P B\left(K_{\beta}+f_{\beta}+\frac{1}{\omega_{n}^{2}} \Delta_{\beta}\left(a_{M \beta}, \dot{a}_{M \beta}, t\right)\right) .
\end{aligned}
$$

Substitute (26) into (27) and consider (14), and we have

$$
\begin{aligned}
\dot{V} \leqslant & -e_{\varepsilon}^{T} Q e_{\varepsilon}-e_{\beta}^{T} Q e_{\beta} \\
& +2 e_{\varepsilon}^{T} P B\left(K_{\varepsilon}+f_{\varepsilon}\right)+2 e_{\beta}^{T} P B\left(K_{\beta}+f_{\beta}\right) \\
& +\frac{2 \bar{a}_{M}}{\omega_{n}^{2}}\left(\left\|P B e_{\varepsilon}\right\|+\left\|P B e_{\beta}\right\|\right) .
\end{aligned}
$$

If we design $K_{\varepsilon}$ and $K_{\beta}$ as follows:

$$
\begin{aligned}
& K_{\varepsilon}=-f_{\varepsilon}-\frac{2 \bar{a}_{M}}{\omega_{n}^{2}} \operatorname{sign}\left(B^{T} P e_{\varepsilon}\right), \\
& K_{\beta}=-f_{\beta}-\frac{2 \bar{a}_{M}}{\omega_{n}^{2}} \operatorname{sign}\left(B^{T} P e_{\varepsilon}\right),
\end{aligned}
$$

then from (28) we have $\dot{V} \leqslant-e_{\varepsilon}^{T} Q e_{\varepsilon}-e_{\beta}^{T} Q e_{\beta}$, which justifies the asymptotic convergence of error vectors $e_{\varepsilon}$ and $e_{\beta}$.

\section{Guidance Law Implementation}

Here we assume that the radar seeker of the missile can provide relative range, relative range rate, and line-of-sight 
angles for guidance law implementation. Since angular rates and accelerations of line-of-sight angles are used in the guidance law, the implementation is focused on numerical differentiation algorithms. Here we employ the following linear differentiator:

$$
\begin{aligned}
& \dot{x}_{1}=x_{2}-\frac{k_{1}}{\varepsilon}\left(x_{1}-f(t)\right), \\
& \dot{x}_{2}=x_{3}-\frac{k_{2}}{\varepsilon^{2}}\left(x_{1}-f(t)\right), \\
& \dot{x}_{3}=-\frac{k_{3}}{\varepsilon^{3}}\left(x_{1}-f(t)\right),
\end{aligned}
$$

where $f(t)$ is the input signal to be differentiated, $\varepsilon>0$ is a small design parameter, and $k_{1}>0, k_{2}>0$, and $k_{3}>0$ are such that

$$
s^{3}+k_{1} s^{2}+k_{2} s+k_{3}
$$

is a Hurwitz polynomial. Here we assume that the third-order derivative of $f$ is bounded; that is, there exists a constant $K_{f}$ such that

$$
\left|f^{(3)}(t)\right| \leqslant K_{f}, \quad \forall t
$$

Define

$$
e=\left[\begin{array}{l}
e_{1} \\
e_{2} \\
e_{3}
\end{array}\right]=\left[\begin{array}{c}
\frac{x_{1}-f(t)}{\varepsilon^{2}} \\
\frac{x_{2}-\dot{f}(t)}{\varepsilon^{2}} \\
x_{3}-\ddot{f}(t)
\end{array}\right],
$$

and from (30) we have

$$
\varepsilon \dot{e}=A_{e} e+\varepsilon B_{e} f^{(3)}(t)
$$

where

$$
A_{e}=\left[\begin{array}{lll}
-k_{1} & 1 & 0 \\
-k_{2} & 0 & 1 \\
-k_{3} & 0 & 0
\end{array}\right], \quad B_{e}=\left[\begin{array}{l}
0 \\
0 \\
1
\end{array}\right]
$$

Since (31) is a Hurwitz polynomial, $A$ is Hurwitz. Therefore, for any given $Q_{e}>0$, there exists a $P>0$, such that

$$
A_{e}^{T} P+P A_{e}=-Q_{e}
$$

Take $V_{e}(e)=e^{T} P_{e} e$, and it is easy to show that system (34) is input-to-state stable [11] with $f^{(3)}(t)$ thought of as an external input, and $\dot{V}(e) \leqslant 0$ whenever $\|e\| \geqslant 2 \varepsilon\left\|P_{e} B_{e}\right\| K_{f} / \lambda_{\text {min }}\left(Q_{e}\right)$. This means that the state of the system (34), the error defined in (33), will converge in finite time $T(\varepsilon)$, dependent on $\varepsilon$, to the following set:

$$
S(\varepsilon)=\left\{e \in \mathbb{R}^{3} \mid\|e\| \leqslant \frac{2 \varepsilon\left\|P_{e} B_{e}\right\| K_{f}}{\lambda_{\min }\left(Q_{e}\right)}\right\},
$$

which is dependent on the parameter $\varepsilon$ and shrinks to zero as $\varepsilon$ tends to zero from above. Thus for an input signal $f$ satisfying (32), the error variables defined in (33) are bounded, and

$$
\begin{aligned}
& \lim _{t \rightarrow \infty, \varepsilon \downarrow 0} x_{1}(t)=f(t), \\
& \lim _{t \rightarrow \infty, \varepsilon \downarrow 0} x_{2}(t)=\dot{f}(t), \\
& \lim _{t \rightarrow \infty, \varepsilon \downarrow 0} x_{3}(t)=\ddot{f}(t) .
\end{aligned}
$$

We can also see from (34) that the converging rates of (38) increase with the decrease of the value of $\varepsilon$ :

$$
\lim _{\varepsilon \downarrow 0} T(\varepsilon)=0
$$

and the bound $K_{f}$ in (32) can be arbitrarily large provided $\varepsilon$ is small enough. However, with the decrease of $\varepsilon$, the error variables $e_{i}(t), i=1,2,3,0<t<T(\varepsilon)$ will become very large, known as the peaking phenomenon [12]. To attenuate the peaking with the set $S(\varepsilon)$ unchanged, we can introduce a satiation function to the differentiator (30). Here the details will not be discussed theoretically, but we note that numerical simulation we conducted has revealed the effectiveness of this technique.

We denote the state of the differentiator (30) by $x_{i}^{\varepsilon}, i=$ $1,2,3$ when the input signal is $q_{\varepsilon}$ and by $x_{i}^{\beta}, i=1,2,3$ when the input signal is $q_{\beta}$. Therefore, the reference signals given in (18) are implemented by replacing $\dot{q}_{\varepsilon}, \ddot{q}_{\varepsilon}, \dot{q}_{\beta}, \ddot{q}_{\beta}$ by $x_{2}^{\varepsilon}, x_{3}^{\varepsilon}$, $x_{2}^{\beta}$, and $x_{3}^{\beta}$, respectively, provided $R, q_{\varepsilon}$, and $q_{\beta}$ are available. As far as measurement noises are concerned, we need to limit the order of differentiation operations as low as possible, especially to avoid very high order differentiations. Here only first-order and second-order differentiations are necessary, and we can limit the effects of noises by limiting the value of the parameter $\varepsilon$. If we used the differentiator to implement $K_{\varepsilon}$ and $K_{\beta}$ in (29), the further differentiation operations on $a_{M \varepsilon r}$ and $a_{M \beta r}$ required in (22) and (23) would have significantly magnified the noises of the measured signals $q_{\varepsilon}$ and $q_{\beta}$, since third-order and fourth-order differentiations are involved. To avoid this, here we give a practical design of $a_{M \varepsilon r}$ and $a_{M \beta r}$, instead of the theoretical design given in (29). The idea is to treat the bounded uncertainties $\Delta_{\varepsilon}$ and $\Delta_{\beta}$ as input signals to dynamics (13) and to reduce the gain of $\Delta_{\varepsilon}$ and $\Delta_{\beta}$ as well as to increase the frequency bandwidths of $a_{M \varepsilon r}$ and $a_{M \beta r}$.

Let

$$
\begin{aligned}
& K_{\varepsilon}=-\frac{2 \zeta(K-1)}{\omega_{n}} \dot{a}_{M \varepsilon}-\left(K^{2}-1\right) a_{M \varepsilon}+\left(K^{2}-1\right) a_{M \varepsilon r}, \\
& K_{\beta}=-\frac{2 \zeta(K-1)}{\omega_{n}} \dot{a}_{M \beta}-\left(K^{2}-1\right) a_{M \beta}+\left(K^{2}-1\right) a_{M \beta r},
\end{aligned}
$$


where $K>1$ is a design parameter, and substitute (40) into (13), and we have

$$
\begin{aligned}
\ddot{a}_{M \varepsilon}= & -2 \zeta\left(K \omega_{n}\right) \dot{a}_{M \varepsilon}-\left(K \omega_{n}\right)^{2} a_{M \varepsilon} \\
& +\left(K \omega_{n}\right)^{2} a_{M \varepsilon r}+\Delta_{\varepsilon}\left(a_{M \varepsilon}, \dot{a}_{M \varepsilon}, t\right), \\
\ddot{a}_{M \beta}= & -2 \zeta\left(K \omega_{n}\right) \dot{a}_{M \beta}-\left(K \omega_{n}\right)^{2} a_{M \beta} \\
& +\left(K \omega_{n}\right)^{2} a_{M \beta r}+\Delta_{\beta}\left(a_{M \beta}, \dot{a}_{M \beta}, t\right) .
\end{aligned}
$$

We can see from (41) that the gains of uncertain input signals $\Delta_{\varepsilon}$ and $\Delta_{\beta}$ are reduced $K^{2}$ times, with the gains of $a_{M \varepsilon r}$ and $a_{M \beta r}$ unchanged and the bandwidths increased $K$ times. Although $K_{\varepsilon}$ and $K_{\beta}$ in (40) cannot guarantee asymptotic convergence of $a_{M \varepsilon}$ and $a_{M \beta}$ to their respective reference signals $a_{M \varepsilon r}$ and $a_{M \beta r}$, as guaranteed by (29), a satisfactory tracking can be guaranteed provided $K$ is sufficiently large.

\section{Simulation}

Here we consider the terminal guidance phase with the following initial conditions:

$$
\begin{array}{ll}
R(0)=6000 \mathrm{~m}, & \dot{R}(0)=600 \mathrm{~m} / \mathrm{s}, \quad q_{\varepsilon}(0)=27^{\circ}, \\
\dot{q}_{\varepsilon}(0)=1.5^{\circ} / \mathrm{s}, & q_{\beta}(0)=30^{\circ}, \quad \dot{q}_{\varepsilon}(0)=-1.25^{\circ} / \mathrm{s} .
\end{array}
$$

The sampling period of the guidance system is assumed to be $T=5 \mathrm{~ms}$. Since the time horizon of a typical terminal guidance phase ranges over a time interval of several seconds or just over ten seconds, we can employ the Euler integration as the numerical implementation of differentiator (30) without much loss of precision; for example,

$$
\begin{aligned}
& x_{1}((k+1) T) \\
& \quad=x_{1}(k T)+T\left[x_{2}(k T)-k_{1} \operatorname{sat}\left(\frac{x_{1}(k T)-f(k T)}{K_{s} \varepsilon}\right)\right], \\
& x_{2}((k+1) T) \\
& \quad=x_{2}(k T)+T\left[x_{3}(k T)-k_{2} \operatorname{sat}\left(\frac{x_{1}(k T)-f(k T)}{K_{s} \varepsilon^{2}}\right)\right], \\
& x_{3}((k+1) T) \\
& \quad=x_{3}(k T)+T\left[-k_{3} \operatorname{sat}\left(\frac{x_{1}(k T)-f(k T)}{K_{s} \varepsilon^{3}}\right)\right],
\end{aligned}
$$

where the parameter $K_{s}$ is set to $80, \varepsilon$ is set to 0.011 , and the parameters $k_{1}, k_{2}$, and $k_{3}$ are set to 3,3 , and 1 , respectively. All the initial values of the differentiator are set to zero. The parameters in the dynamics (13) are set to $\zeta=0.7$ and $\omega_{n}=8$. The maximum acceleration the missile can provide is assumed to be $\bar{a}_{M}=200 \mathrm{~m} / \mathrm{s}^{2}$, and the

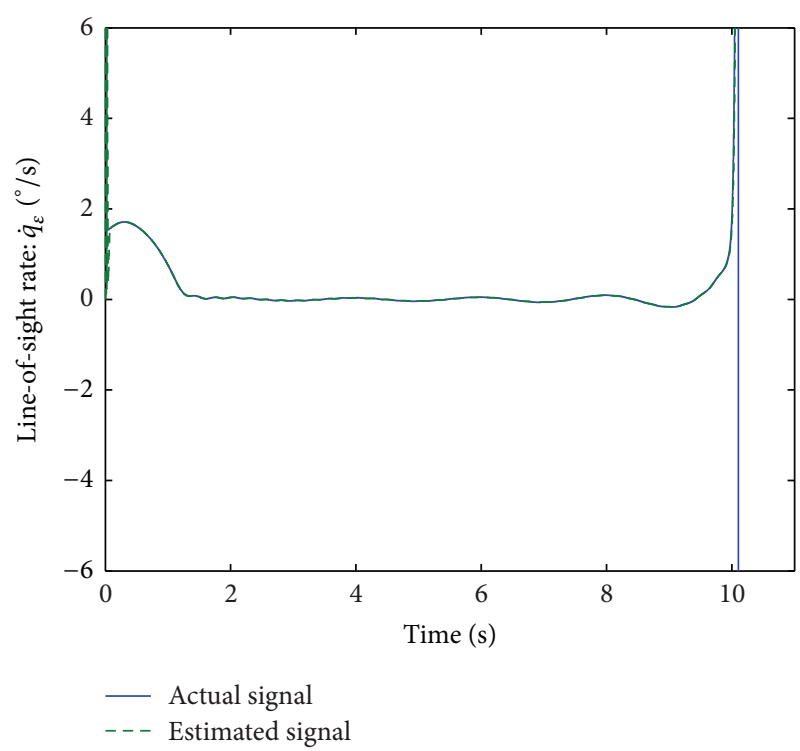

FIGURE 2: Line-of-sight rate $\dot{q}_{\varepsilon}$ and its estimate: without noises.

commanded accelerations issued by the guidance system take the following form:

$$
\begin{aligned}
& a_{M \varepsilon c}=\bar{a}_{M} \operatorname{sat}\left(\frac{a_{M \varepsilon r}+K_{\varepsilon}}{\bar{a}_{M}}\right), \\
& a_{M \beta c}=\bar{a}_{M} \operatorname{sat}\left(\frac{a_{M \beta r}+K_{\beta}}{\bar{a}_{M}}\right),
\end{aligned}
$$

where $a_{M \varepsilon r}$ and $a_{M \beta r}$ are given in (18) with $k=5$, and $K_{\varepsilon}$ and $K_{\beta}$ are given in (40) with $K=4$. The minimum range for the seeker to operate is assumed to be $\bar{R}_{b}=100 \mathrm{~m}$, and when $R<\bar{R}_{b}$, both $a_{M \varepsilon c}$ and $a_{M \beta c}$ are set to zero.

Numerical simulation is conducted for the above scenario with an integration step $0.0001 \mathrm{~s}$. For a nonmaneuvering target, the interception time is $10.0577 \mathrm{~s}$, and the miss distance is $0.0475 \mathrm{~m}$. Then we consider the case where the maneuver of the target takes the following form:

$$
\begin{aligned}
& a_{T \varepsilon}=100 \sin \left(2 \pi f_{\varepsilon}+\theta_{\varepsilon}\right) \mathrm{m} / \mathrm{s}^{2}, \\
& a_{T \beta}=100 \sin \left(2 \pi f_{\beta}+\theta_{\beta}\right) \mathrm{m} / \mathrm{s}^{2},
\end{aligned}
$$

where $f_{\varepsilon}=0.35 \mathrm{~Hz}, \theta_{\varepsilon}=\pi / 5, f_{\beta}=0.5 \mathrm{~Hz}$, and $\theta_{\beta}=\pi / 5$. The interception time is $10.1039 \mathrm{~s}$, and the miss distance is $0.1343 \mathrm{~m}$. The line-of-sight rates $\dot{q}_{\varepsilon}$ and $\dot{q}_{\beta}$ and their respective estimates are shown in Figures 2 and 3 , and a satisfactory tracking is obtained. The acceleration components of the missile are shown in Figure 4.

To validate the proposed guidance law and its implementation when measurement is corrupted by noises, the simulation is also conducted with $q_{\varepsilon}$ and $q_{\beta}$ added with a normal stochastic noise with zero mean and standard deviation $\sigma=30 \mu \mathrm{rad}$. Numerical simulation is repeated for 100 times. The miss distance values, with a mean value of $0.4856 \mathrm{~m}$ and a standard deviation of $0.3372 \mathrm{~m}$, are shown in Figure 5. The line-of-sight rates $\dot{q}_{\varepsilon}$ and $\dot{q}_{\beta}$ and their respective 


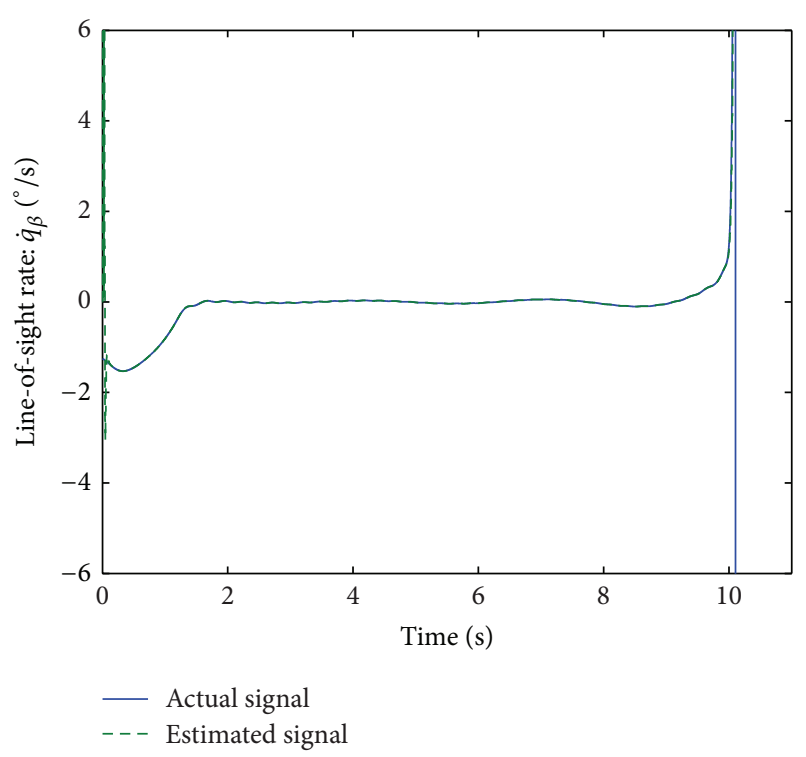

FiguRE 3: Line-of-sight rate $\dot{q}_{\beta}$ and its estimate: without noises.

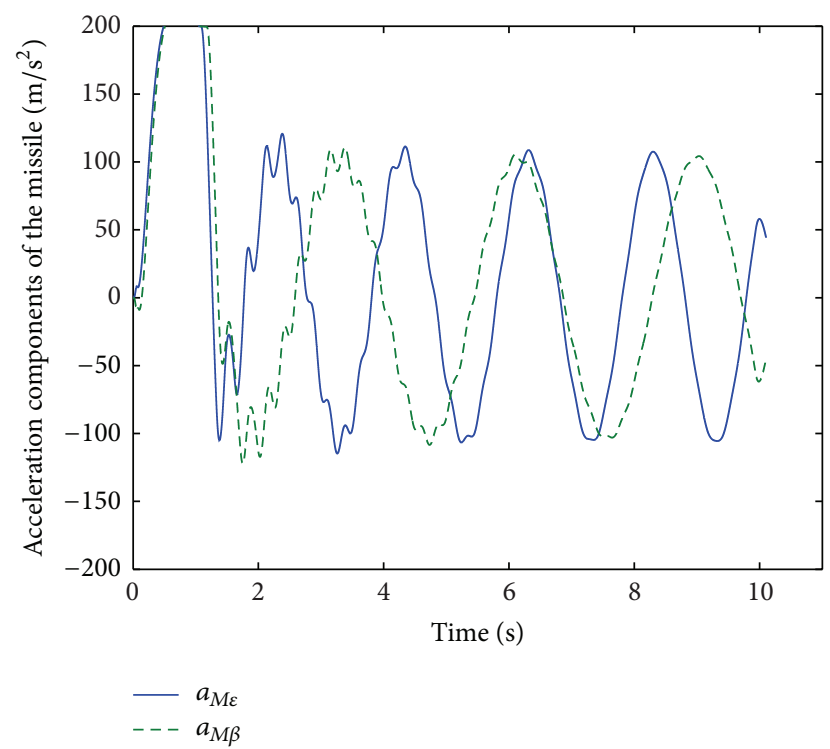

FIGURE 4: The acceleration components of the missile: without noises.

estimates under the noisy condition are shown in Figures 6 and 7. The lateral acceleration components of the missile are shown in Figure 8.

\section{Conclusion}

A novel terminal guidance law design and its implementation are considered for missiles against maneuvering targets. The target's maneuvers are compensated for via feeding back the line-of-sight accelerations. Therefore no maneuver models for the targets are necessary. The lateral acceleration dynamics are also incorporated into the guidance commands. The

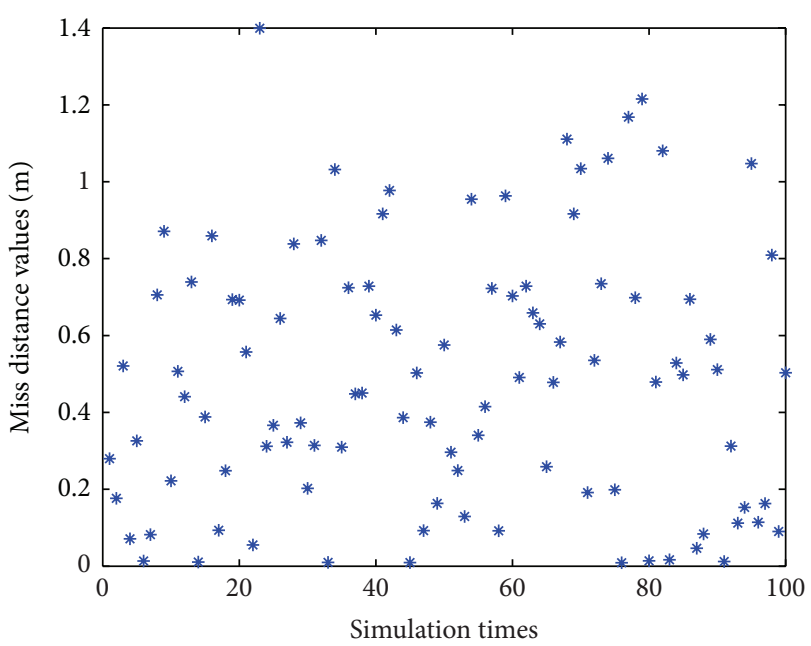

Figure 5: The miss distance values: with noises.

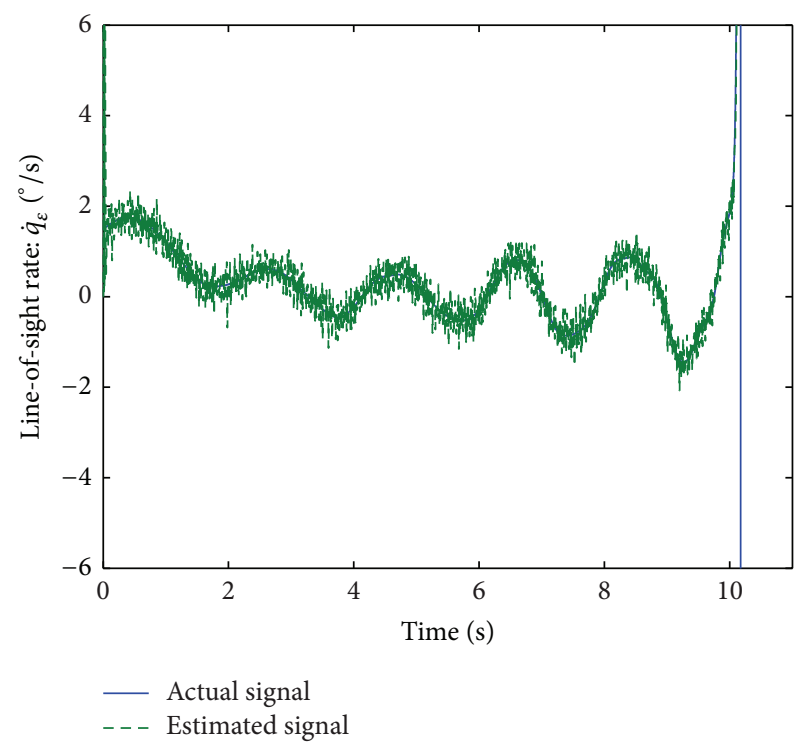

FIGURE 6: Line-of-sight rate $\dot{q}_{\varepsilon}$ and its estimate: with noises.

implementation of the guidance laws only requires the firstorder and second-order derivative signals of the line-ofsight angles. A high-gain linear differentiator is used in the guidance law implementation. Numerical simulation is conducted to validate the design and implementation. In the simulation, both cases with and without measurement noises are considered. The results show the effectiveness. We note that, in the implementation, the differentiator employs no statistical features of the noises, which deserves further investigation to improve the performance of the given design.

\section{Conflict of Interests}

The authors declare that there is no conflict of interests regarding the publication of this paper. 


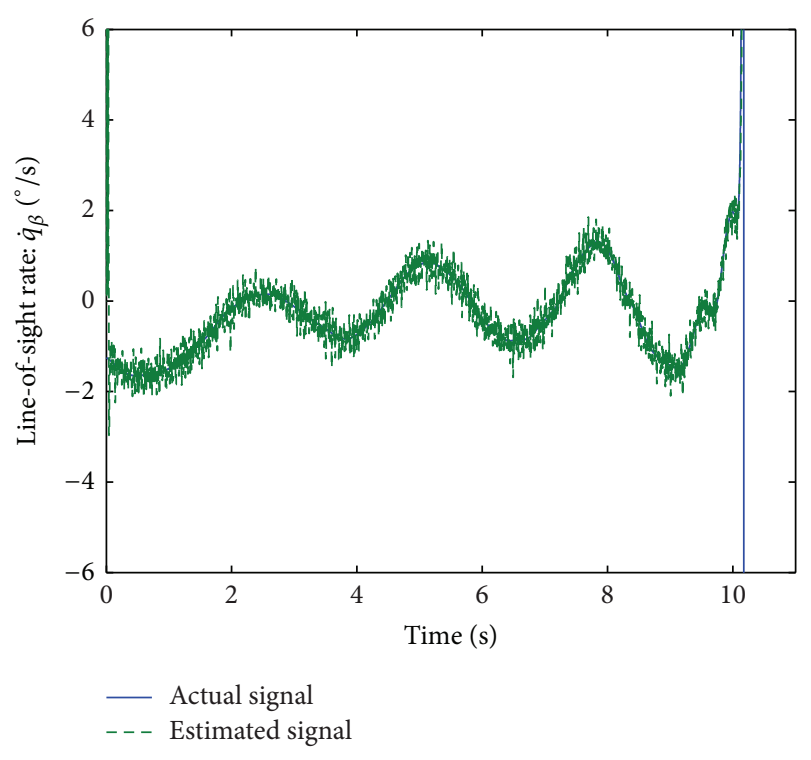

FIGURE 7: Line-of-sight rate $\dot{q}_{\beta}$ and its estimate: with noises.

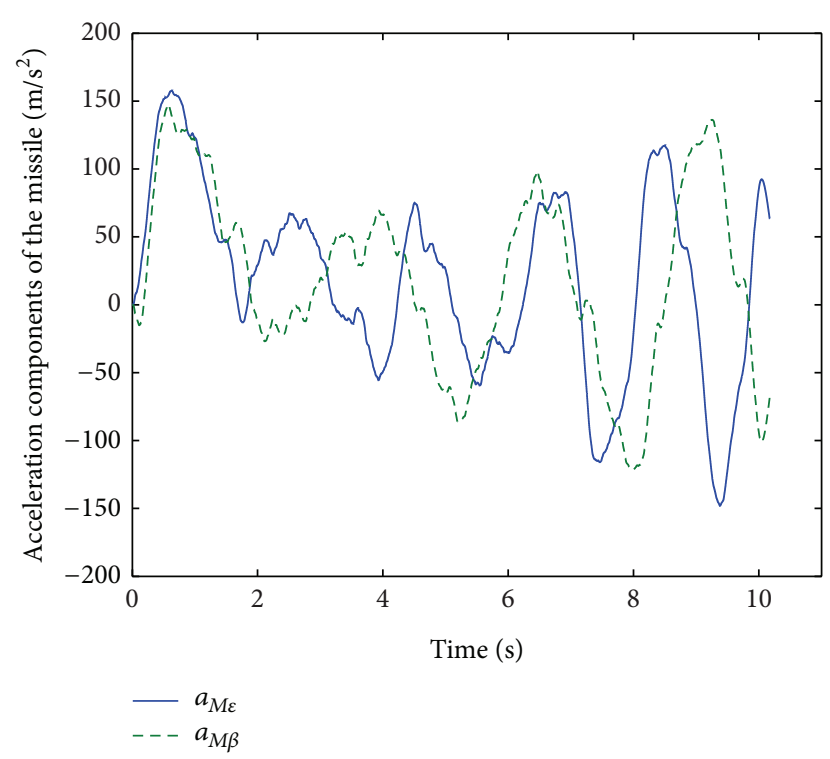

FIGURE 8: The acceleration components of the missile: with noises.

\section{Acknowledgments}

This work was supported in part by the National Natural Science Foundation of China under Grant nos. 61174001, 61203185 , and 61321062. The work of the second author was partially supported by the Natural Science Foundation of Heilongjiang Province of China (no. F201221) and the Graduate Education Innovation Project of Heilongjiang Province of China (no. JGXM_HLJ_2013034).

\section{References}

[1] G. Siouris, Missile Guidance and Control Systems, Springer, New York, NY, USA, 2003.
[2] P. Zarchan, Tactical and Strategic Missile Guidance, AIAA, Reston, Va, USA, 3rd edition, 2012.

[3] X. Ku and V. Jilkov, "A survey of maneuvering target tracking. Part I. Dynamic models," IEEE Transactions on Aerospace and Electronic Systems, vol. 39, no. 4, pp. 1333-1364, 2003.

[4] K. Ma, H. K. Khalil, and Y. Yao, "Guidance law implementation with performance recovery using an extended high-gain observer," Aerospace Science and Technology, vol. 24, no. 1, pp. 177-186, 2013.

[5] Z. Zhu, D. Xu, J. Liu, and Y. Xia, "Missile guidance law based on extended state observer," IEEE Transactions on Industrial Electronics, vol. 60, no. 12, pp. 5882-5891, 2013.

[6] B. S. Kim, J. G. Lee, H. S. Han et al., "Homing guidance with terminal angular constraint against nonmaneuvering and maneuvering targets," in Proceedings of the AIAA Guidance, Avigation, and Control Conference, AIAA-97-3474, pp. 189-199, 1997.

[7] H. Yanhua and X. Bo, "Variable structure guidance law for attacking surface maneuver targets," Journal of Systems Engineering and Electronics, vol. 19, no. 2, pp. 337-341, 2008.

[8] Y. S. Choi, H. C. Lee, and J. W. Choi, "Autopilot design for agile missile with aerodynamic fin and side thruster," in Proceedings of the SICE Annual Conference, vol. 2, pp. 1476-1481, Fukui, Japan, August 2003.

[9] L. P. Jeffrey and J. R. Christopher, "A comparison of turbulence models for a supersonic jet in transonic crossflow," in Proceedings of the 39th AIAA Aerospace Sciences Meeting and Exhibit, pp. 1-12, Reno, Nev, USA, 2001.

[10] N. F. Palumbo, B. E. Reardon, and R. A. Blauwkamp, "Integrated guidance and control for homing missiles," Johns Hopkins Applicaiton Technical Digest, vol. 25, no. 2, pp. 121-138, 2004.

[11] H. K. Khalil, Nonlinear Systems, Prentice Hall, Upper Saddle River, NJ, USA, 3rd edition, 2002.

[12] H. K. Khalil, "High-gain observers in nonlinear feedback control," in Proceedings of the International Conferene on Control, Automation and Systems, pp. 249-268, Seoul, Republic of Korea, 2008. 


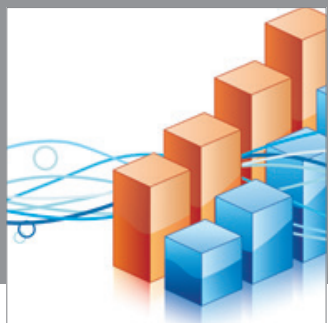

Advances in

Operations Research

mansans

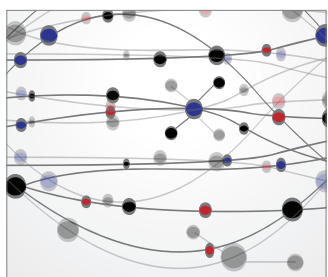

The Scientific World Journal
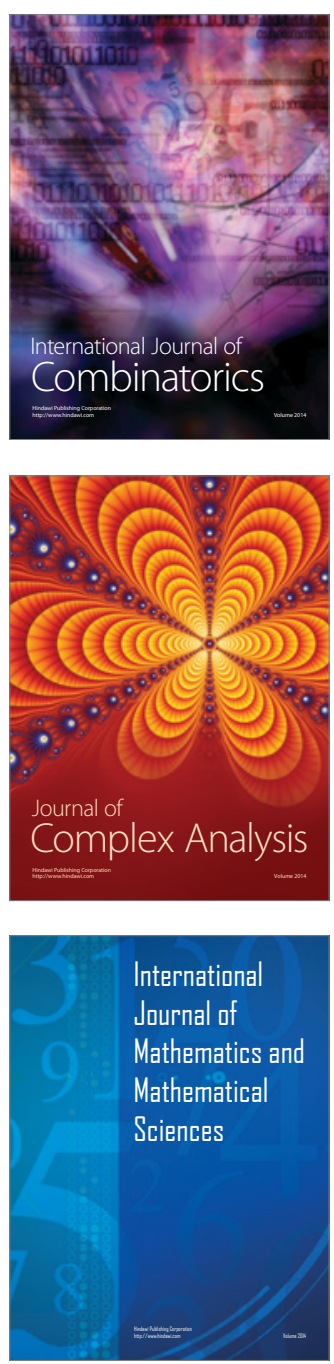
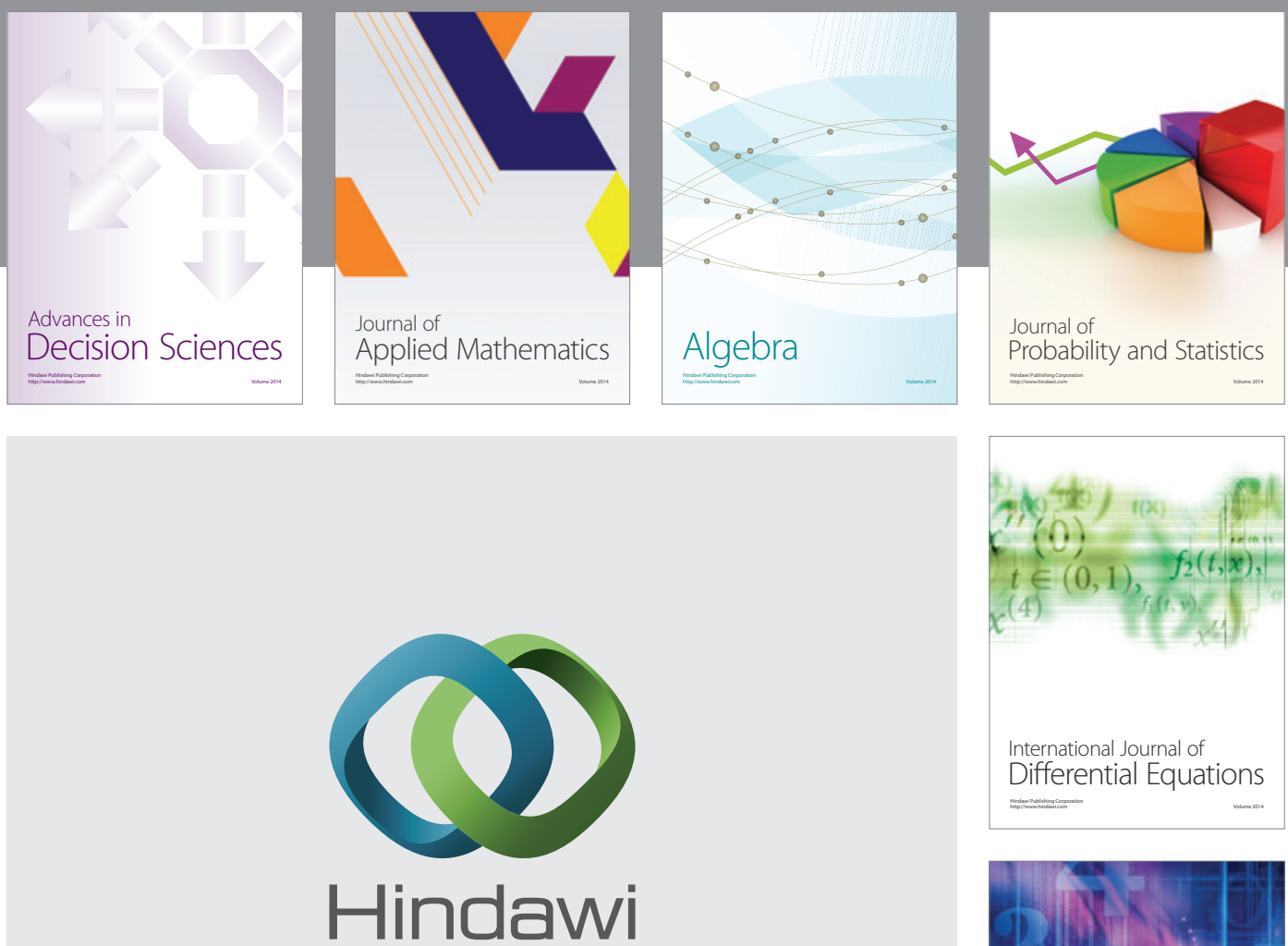

Submit your manuscripts at http://www.hindawi.com
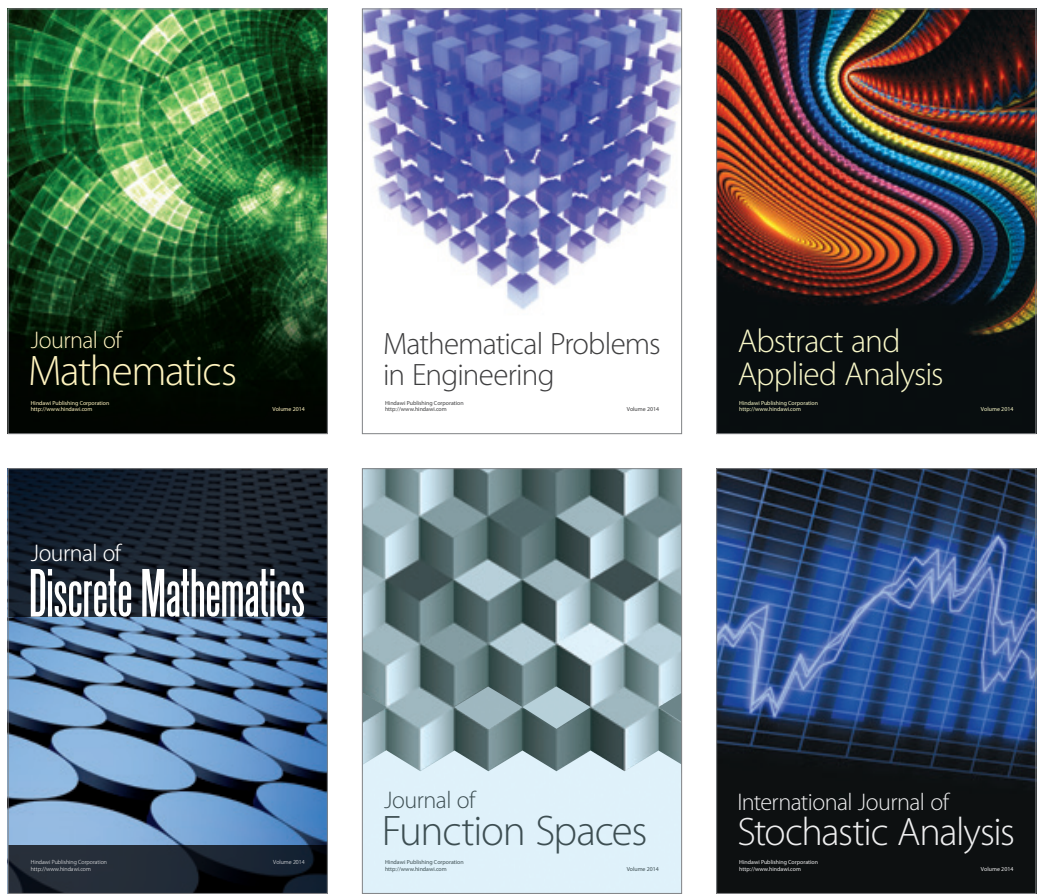

Journal of

Function Spaces

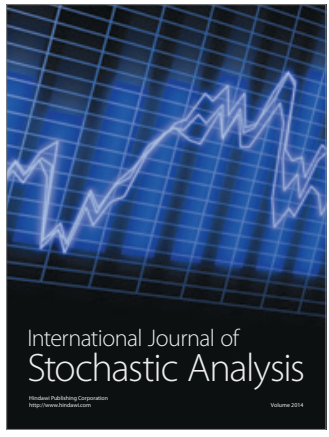

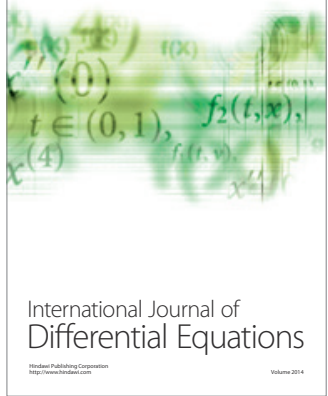
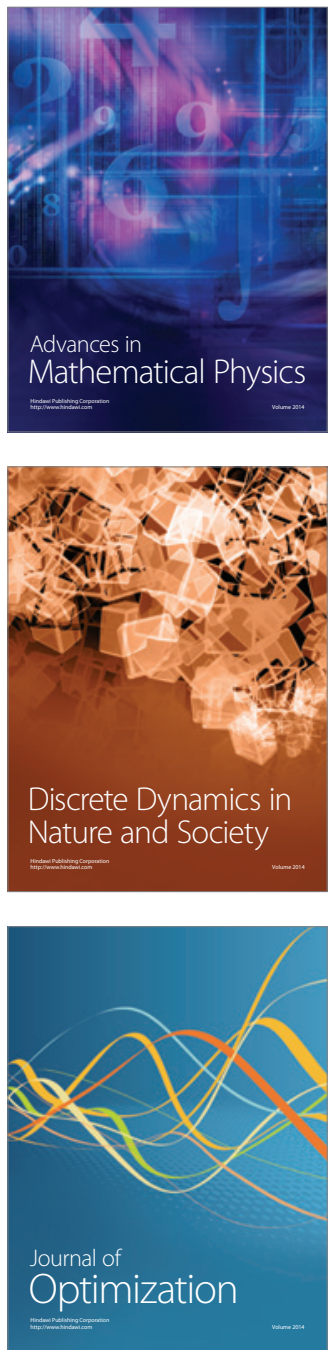